\title{
Low-Complexity Linear Equalization for Block Transmission in Multipath Channels
}

\author{
Shakti Prasad Shenoy ${ }^{\dagger *}$, Francesco Negro*, Irfan Ghauri*, Dirk T.M. Slock ${ }^{\dagger}$ \\ *Infineon Technologies France SAS, GAIA, 2600 Route des Crêtes, 06560 Sophia Antipolis Cedex, France \\ Email: shakti.shenoy@eurecom.fr, francesco.negro@infineon.com, irfan.ghauri@infineon.com \\ ${ }^{\dagger}$ Mobile Communications Department, Institut Eurécom, 2229 Route des Crêtes, 06904 Sophia Antipolis Cedex, France \\ Email: dirk.slock@eurecom.fr
}

\begin{abstract}
We address the issue of low complexity linear equalization for cyclic-prefix (CP) and zero-padded (ZP) block transmissions (OFDM). While cyclic-prefix (CP) OFDM does not exploit frequency diversity offered by multipath fading, use of appropriate redundant linear precoding permits a linear equalizer ( $\mathrm{LE}$ ) to benefit from full diversity in the frequency selective channel [1]. We exploit excess time of block transmission (TX) for low-complexity (and full-diversity) linear equalization. Relying on specific structure in the TX scheme, it is shown that excess time can take different forms and impacts diversity order of linear (block) equalization at the receiver (RX). We focus on frequency selective channels and revisit full-diversity lowcomplexity LE for the ZP case. We discuss special cases of ZPOFDM with excess time that admit full-diversity LE and draw parallels with the standard $\mathrm{ZP}$ case.
\end{abstract}

Index Terms-Block Transmission, Diversity Order, Linear Equalization, Fading Channels

\section{INTRODUCTION}

Frequency selective fading provides multipath diversity due to the presence of multiple independently fading components. OFDM converts the frequency selective channel into independent flat-fading parallel channels corresponding to the number of subcarriers in the OFDM system. CP-OFDM is based on the principle that $\mathrm{CP}$ imposes a circulant structure on the resultant channel matrix. The IDFT and DFT transform pair used to separate the broadband transmission into parallel narrowband channels effectively diagonalises this channel thus paving the way for simple equalization techniques followed by symbolby-symbol detection. The price paid for low complexity signal processing is a complete loss of multipath diversity.

In CP-OFDM frequency diversity does not get exploited with LE. Redundant linear precoding however remedies this completely as shown in [1]. A linear precoder is said to be of redundancy $p$ if it mixes $N-p$ OFDM symbols over $N$ tones. It has been shown in [1] that as long as the precoding introduces redundancy greater than the maximum degree of singularity $L$ that the channel can suffer (without becoming completely zero), the LE allows full diversity. Indeed, the FIR SISO channel can show zeros at most on $L$ tones. If more zeros would appear, then the whole channel impulse response is zero. ZP, being an instance of linear-precoding, achieves fulldiversity with LE, where the Toeplitz structure of the channel matrix always guarantees full column rank until the entire channel response is zero! In practical systems, the problem is the complexity of LE that involves inverses of matrices of degree $N$. [2] proposes low-complexity solutions, among which one at the expense of full-diversity.

In this paper, we study the performance of linear equalizers for CP-OFDM and ZP-OFDM over frequency selective channels. In the first instance, we draw attention to the relationship between LE for ZP-OFDM and for CP-OFDM. With this relation, we are able to obtain a low-complexity implementation of full-diversity LE for ZP-OFDM. Excesstime in block TX is the extra time available for TX of an (OFDM) symbol across the channel. This is usually a guardtime (cyclic prefix longer than channel length) or trailing zeros as in the case of ZP-OFDM where the tail coefficients of the channel allow the signal to taper-off and thus avoid inter-symbol interference. We show that while excess time in ZP leads to the desirable diversity benefit, excess time in improves performance (coding gain) in $\mathrm{CP}$ but does not provide diversity order benefits. It is shown that a special case of ZP-OFDM that exploits knowledge of channel length at TX enables low-complexity and full-diversity linear equalization at the receiver.

\section{Signal Model}

Consider a $N$-subcarrier OFDM system. At the transmitter, complex-modulated symbols are parsed into a $N$-length complex symbol vector $\mathbf{s}=[s(0), s(1), \ldots, s(N-1)]^{T}$ consisting of symbols that are to be transmitted on $N$ subcarriers. After passing through an IDFT block, depending on the transmission scheme, a guard period of length $G \geq L$ is either pre-pended or post-pended to the signal to obtain a $P$-length OFDM symbol that can be expressed as

$$
\mathbf{x}=\mathbf{C} \mathbf{F}^{H} \mathbf{s}
$$

where $P=(N+G), L$ is the maximum delay spread of the channel, $\mathbf{F}$ is the standard $N \times N$ Fourier matrix whose entries are given by $[\mathbf{F}]_{n, k}=e^{-j 2 \pi k n / N}$ and $\mathbf{C}$ is a $P \times N$ matrix that represents an appropriate guard period insertion before transmission. Let $\mathbf{H}_{t}$ denote the Toeplitz structured channel matrix in time domain with $\left[h_{0}, h_{1}, \ldots, h_{L-1}, \mathbf{0}_{(1 \times P-L)}\right]^{T}$ as its first column. Then, in general, at the receiver, the equivalent channel matrix $\mathbf{H}_{e q}$ can be expressed as

$$
\mathbf{H}_{e q}=\mathbf{D H}_{t} \mathbf{C}
$$


The matrix pair $\mathbf{C}$ and $\mathbf{D}$ together determine the dimensions and properties of the equivalent channel matrix (and hence the received signal) based on a given transmission scheme.

For the CP-OFDM case, $\mathbf{C}$ is a $P \times N$ matrix that represents the operation of $\mathbf{C P}$ insertion at the transmitter and $\mathbf{D}$ is a $N \times P$ matrix that represents full $\mathrm{CP}$ removal at the receiver. Their combined effect is to render $\mathbf{H}_{e q}$ circulant. In the case of $\mathrm{ZP}-\mathrm{OFDM}, \mathbf{C}$ is a $P \times N$ matrix that represents the operation of ZP post-fix to $\tilde{\mathbf{s}}=\mathbf{F}^{H} \mathbf{S}$ and $\mathbf{D}$ is simply an identity matrix $\mathbf{I}_{P}$. The effect of the matrix pair here is to completely remove inter-block-interference (IBI) in the received block while also promising invertibility of $\mathbf{H}_{e q}$. We can now readily represent the input signal at the receiver due to the $j^{\text {th }}$ transmit block as

$$
\mathbf{r}[j]=\mathbf{D H}_{t} \mathbf{C F}^{H} \mathbf{s}[j]+\mathbf{v}[j],
$$

where $\mathbf{s}[j]=[s(j N+0), s(j N+1), \ldots, s(j N+N-1)]^{T}$ and $\mathbf{v}[j]$ is a zero-mean AWGN vector whose entries have variance $\sigma_{v}^{2}$.

\section{LOW COMPLEXITY LINEAR EQUALIZATION}

In this section we discuss multipath diversity of lowcomplexity linear equalizers in frequency selective channels. Since we consider block transmission schemes, we omit the block index $j$ since processing is on a block-by-block basis.

\section{A. Zero-Padded OFDM}

For ZP-OFDM the $\mathbf{C}$ matrix in Eq. (2) is denoted as $\mathbf{C}_{z p}$ and is expressed as $\mathbf{C}_{z p}=\left[\begin{array}{ll}\mathbf{I}_{N} & \mathbf{0}\end{array}\right]^{T}$ where $\mathbf{0}$ is a $N \times G$ allzero sub-matrix. The received block symbol in Eq. (3) is then represented as

$$
\mathbf{r}_{z p}=\mathbf{H}_{z p} \mathbf{F}^{H} \mathbf{s}+\mathbf{v},
$$

where we first partition the channel matrix as $\mathbf{H}_{t}=\left[\mathbf{H}_{z p} \tilde{\mathbf{H}}\right]$ with the $P \times N$ sub-matrix $\mathbf{H}_{z p}$ representing the IBI free channel matrix and the $P \times G$ block $\tilde{\mathbf{H}}$ representing the channel submatrix that produces IBI due to the previous transmit block. Since this $\mathbf{H}$ multiplies the all zero sub-matrix of $\mathbf{C}_{z p}$ the received signal is represented as in Eq. (4). $\mathbf{H}_{z p}$ is thus a $P \times N$ banded Toeplitz matrix with $\left[h_{0}, h_{1}, \ldots, h_{L-1}, \mathbf{0}_{(1 \times P-L)}\right]^{T}$ as its first column. For single carrier-ZP, and it can be shown that

$$
\begin{aligned}
\mathbf{r}_{s c} & =\mathbf{H}_{z p} \mathbf{s}+\mathbf{v}, \\
\mathbf{H}_{z p} & =\mathbf{F}_{P}^{H} \mathcal{D} \boldsymbol{\Phi},
\end{aligned}
$$

where $\mathbf{F}_{P}$ is a DFT matrix with $\left[\mathbf{F}_{P}\right]_{n, k}=(1 / \sqrt{P}) e^{-j 2 \pi k n / P}$, $\mathcal{D}$ is the resultant diagonal channel matrix in frequency domain and $\Phi$ can be interpreted as a precoding matrix composed of the first $N$ columns of $\mathbf{F}_{P}$ thus having a Vandermonde structure. However, for ZP-OFDM,

$$
\mathbf{F}_{P} \mathbf{r}_{z p}=\mathbf{F}_{P}^{H} \mathcal{D} \mathbf{V} \mathbf{s}+\mathbf{F}_{P} \mathbf{v},
$$

yields a similar interpretation as a precoded OFDM system in [1] but with a precoding matrix $\mathbf{V}$ such that $[\mathbf{V}]_{p, q}=$ $N-1$ $\sum_{k=0}^{N-1} e^{-j 2 \pi k(p / P-q / N)}$.

At first glance, the MMSE-ZF equalizer $\left(\mathbf{H}_{z p}^{H} \mathbf{H}_{z p}\right)^{-1} \mathbf{H}_{z p}^{H}$ that achieves full diversity for ZP-OFDM seems to have a huge computational complexity due to the large matrix inversion problem. However, a simple yet insightful observation leads to reduced complexity implementation of MMSE-ZF equalizer for ZP-OFDM. Compare the channel matrix of CP-OFDM with $N+L$ subcarriers and a cyclic prefix of length $L$, with that of a ZP-OFDM system with $P=N+L$. After CP removal the equivalent channel matrix for CP-OFDM is a $P \times P$ circulant matrix $\mathbf{H}_{c p}$ with the $P \times N$ banded Toeplitz matrix $\mathbf{H}_{z p}$ representing the equivalent channel matrix of the ZP-OFDM system with $P=N+L$ embedded in it. In other words we can partition $\mathbf{H}_{c p}$ as $\mathbf{H}_{c p}=\left[\begin{array}{ll}\mathbf{H}_{z p} & \mathbf{H}_{0}\end{array}\right]$. We know that $\overline{\mathbf{F}} \mathbf{H}_{c p} \overline{\mathbf{F}}^{H}=\boldsymbol{\Lambda}$ is a diagonal matrix. $\overline{\mathbf{F}}$ is the associated normalized $(N+L)$-DFT matrix. From the above relation, we have

$$
\begin{aligned}
\overline{\mathbf{F}}^{H}\left(\boldsymbol{\Lambda}^{H} \boldsymbol{\Lambda}\right)^{-1} \overline{\mathbf{F}} & =\left(\mathbf{H}_{c p}^{H} \mathbf{H}_{c p}\right)^{-1} \\
{\left[\begin{array}{cc}
\mathcal{A} & \mathcal{B} \\
\mathcal{B}^{H} & \mathcal{C}
\end{array}\right]^{-1} } & =\left[\begin{array}{ll}
\mathbf{H}_{z p}^{H} \mathbf{H}_{z p} & \mathbf{H}_{z p}^{H} \mathbf{H}_{0} \\
\mathbf{H}_{0}^{H} \mathbf{H}_{z p} & \mathbf{H}_{0}^{H} \mathbf{H}_{0}
\end{array}\right]^{-1}
\end{aligned}
$$

The block matrix of the RHS of the equation can be inverted using the following identity [3]

$$
\left[\begin{array}{cc}
\mathcal{A}^{-1}+\mathcal{E} \Delta^{-1} \mathcal{E}^{H} & -\mathcal{E} \Delta^{-1} \\
-\boldsymbol{\Delta}^{-1} \mathcal{E}^{H} & \boldsymbol{\Delta}^{-1}
\end{array}\right]
$$

where $\boldsymbol{\Delta}=\mathcal{C}-\mathcal{B}^{H} \mathcal{A}^{-1} \mathcal{B}$ and $\mathcal{E}=\mathcal{A}^{-1} \mathcal{B}$. By first constructing $\overline{\mathbf{F}}^{H}\left(\boldsymbol{\Lambda}^{H} \boldsymbol{\Lambda}\right)^{-1} \overline{\mathbf{F}}$ and extracting the appropriate matrix blocks corresponding to $\mathcal{Q}=\mathcal{A}^{-1}+\mathcal{E} \Delta^{-1} \mathcal{E}^{H}$, $-\mathcal{E} \Delta^{-1}, \boldsymbol{\Delta}^{-1}$ and $\boldsymbol{\Delta} \mathcal{E}^{H}$ the MMSE-ZF equalizer can be reconstructed as

$$
\left(\mathbf{H}_{z p}^{H} \mathbf{H}_{z p}\right)^{-1} \mathbf{H}_{z p}^{H}=\left(\mathcal{Q}-\mathcal{E} \boldsymbol{\Delta}^{-1} \cdot \boldsymbol{\Delta} \cdot \boldsymbol{\Delta}^{-1} \mathcal{E}^{H}\right) \mathbf{H}_{z p}^{H},
$$

The total effort required for matrix inversion is thus reduced to inversion of the diagonal matrix $\left(\boldsymbol{\Lambda}^{H} \boldsymbol{\Lambda}\right)$ and the $L \times L$ matrix $\boldsymbol{\Delta}^{-1}$ thereby significantly reducing the complexity of MMSE-ZF equalizer.

1) Discussion: In [4] an alternative closed form expression for MMSE-ZF equalizer is presented for the ZP case. The equalizer proposed by the author consists of two terms. The first corresponds to equalization similar to that for singlecarrier cyclic prefix (SC-CP) while the second term can be interpreted as a correction term for ZP. Ignoring addition operations, the computational complexity of this equalizer is slightly greater than the equalizer implementation we propose here since it requires $2 P$ more multiplications and one $\mathcal{O}(P \log L)$ operation more than our proposed implementation.

\section{B. Cyclic Prefixed ZP-OFDM}

We now introduce a new variation of ZP-OFDM with the goal of achieving full multipath diversity with low-complexity linear equalization at the receiver. The scheme exploits knowledge of channel length at the transmitter to split the available guard interval into a cyclic prefixed-interval and a zero-padded postfix instead of relegating the entire guard interval to a cyclic prefix or trailing zeros. Consider a guard interval $G>L$ available at the transmitter, after the N-point IDFT, a $L$ length cyclic prefix is added to $\tilde{\mathbf{s}}=\mathbf{F}^{H} \mathbf{s}$. The resultant 
$N+L$ length vector is then extended to $P$ by appending $e_{t}=P-N-L$ zeros. We will see later in the section that it is precisely this excess time that allows the receiver to achieve full diversity. The post-IDFT processing at the transmitter can then be represented by the block matrix $\mathbf{C}_{z c}=\left[\begin{array}{ll}\mathbf{C}_{c p} & \mathbf{0}\end{array}\right]^{T}$. Where $\mathbf{C}_{c p}$ is $L$-length $\mathrm{CP}$ insertion matrix and $\mathbf{0}$ is an all zero matrix with $e_{t}$ rows. This leads us to the transmit signal representation

$$
\mathbf{x}=\mathbf{C}_{z c} \mathbf{F}^{H} \mathbf{s},
$$

At the receiver, after discarding the $L$-length $\mathrm{CP}$ of the received signal $\mathbf{r}$, the $N+e_{t}$-length input $\tilde{\mathbf{y}}$ to the equalizer can be represented as

$$
\widetilde{\mathbf{y}}=\mathbf{D}_{z c} \mathbf{H}_{t} \mathbf{C}_{z c} \mathbf{F}^{H} \mathbf{S}+\tilde{\mathbf{v}},
$$

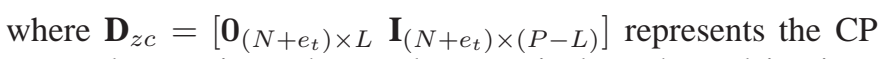
removal operation. The resultant equivalent channel is given by

$$
\mathbf{H}_{z c}=\left[\begin{array}{cccccc}
h_{0} & 0 & \cdots & h_{L-1} & \cdots & h_{1} \\
h_{1} & h_{0} & \cdots & \cdots & \cdots & h_{2} \\
\vdots & \vdots & \cdots & \cdots & \cdots & \cdots \\
\vdots & \vdots & \vdots & \cdots & \cdots & h_{L-1} \\
h_{L-1} & h_{L-2} & \cdots & 0 & \cdots & 0 \\
0 & \ddots & \ddots & \ddots & \ddots & 0 \\
0 & \ddots & h_{L-1} & \ddots & \ddots & h_{0} \\
0 & \ddots & \ddots & \ddots & \ddots & \ddots \\
0 & 0 & 0 & 0 & 0 & h_{N+e_{t}-1}
\end{array}\right]
$$

and can be represented as a block matrix $\mathbf{H}_{z c}=\left[\begin{array}{ll}\mathbf{H}_{c} & \mathbf{H}_{e}\end{array}\right]^{T}$. $\mathbf{H}_{c}$ is a square circulant matrix with $N$ rows and $\mathbf{H}_{e}$ is the time-domain channel matrix corresponding to the excess time and is sparse upper-triangular Toeplitz matrix of dimension $e_{t} \times N . \widetilde{\mathbf{y}}$ can equivalently be represented as

$$
\widetilde{\mathbf{y}}=\left[\begin{array}{c}
\widetilde{\mathbf{y}} \\
\mathbf{y}_{e}
\end{array}\right]=\left[\begin{array}{c}
\mathbf{H}_{c} \\
\mathbf{H}_{e}
\end{array}\right] \mathbf{F}^{H} \mathbf{s}+\left[\begin{array}{c}
\widetilde{\widetilde{\mathbf{v}}} \\
\widetilde{\mathbf{v}}_{e}
\end{array}\right],
$$

We now note that $\mathbf{H}_{c}$ is diagonalizable by an IDFT operation. The reason for diagonalization is simple. Equalization at this stage involves, among other things, inversion of $\mathbf{H}_{z c}^{H} \mathbf{H}_{z c}$ which is computationally intensive. Transformation of $\mathbf{H}_{c}$ with DFT on the other hand lends a unique structure to the channel matrix making it a predominantly diagonal matrix. Standard linear algebra then reduces the computational complexity of the resultant matrix inversion to a very low order. With this in mind, we process $\widetilde{\mathbf{y}}$ with a DFT while retaining $\mathbf{y}_{e}$ as is. Representing this operation by $\mathbf{J}$, we arrive at the following equivalent receive signal representation.

$$
\begin{aligned}
\mathbf{y} & =\mathbf{J} \mathbf{y} \\
& =\left[\begin{array}{c}
\mathbf{F H}_{c} \mathbf{F}^{H} \\
\mathbf{H}_{e} \mathbf{F}^{H}
\end{array}\right] \mathbf{s}+\mathbf{v} \\
& =\left[\begin{array}{c}
\mathcal{D} \\
\mathcal{H}_{e}
\end{array}\right] \mathbf{s}+\mathbf{v}=\mathcal{H} \mathbf{s}+\mathbf{v}
\end{aligned}
$$

The MMSE-ZF equalizer in this case is given by $\mathbf{G}=$ $\left(\mathcal{H}^{H} \mathcal{H}\right)^{-1} \mathcal{H}^{H}$. The pre-processing pay-off now starts to become obvious. Note that $\mathcal{H}$ is a rank- $e_{t}$ modification to a strictly diagonal $\mathcal{D}$. The inversion effort of this matrix can therefore be reduced significantly by expressing it in the form

$$
\left(\mathcal{H}^{H} \mathcal{H}\right)^{-1}=\left(\mathcal{H}_{e}^{H} \mathcal{H}_{e}+\mathcal{D}^{H} \mathcal{D}\right)^{-1},
$$

Let $A=\mathcal{D}^{H} \mathcal{D}, B=\mathcal{H}_{e}^{H}, C=\mathbf{I}_{e_{t}}$ and $D=\mathcal{H}_{e}$. Now, applying the Matrix Inversion Lemma ${ }^{1}$, the matrix inversion effort is reduced to that of inverting an $e_{t} \times e_{t}$ matrix $\left(C^{-1}+D A^{-1} B\right)^{-1}$. Though this involves an inversion of $A$, this inversion is also of low complexity since it is a diagonal matrix. Thus complexity of the equalizer is significantly reduced. The fact that linear equalization in this case achieves full diversity is explained by the "triangular" structure of $\mathbf{H}_{e}$ which ensures full column rank of the matrix and thus provides full diversity. Assuming input symbols are white with variance $\sigma_{a}^{2}$, one can also do a similar treatment for the MMSE estimator. The MMSE estimator for the transmit signal $\mathbf{s}$ given $\mathbf{y}$ is then given by $\mathbf{R}_{s y} \mathbf{R}_{y y}^{-1}$ and the equalizer can be expressed as $\mathbf{G}_{\text {mmse }}=\left(\mathcal{H}^{H} \mathcal{H}+\sigma_{v}^{2} / \sigma_{a}^{2} \mathbf{I}\right)^{-1} \mathcal{H}^{H}$.

\section{C. $C P$-OFDM}

Consider again a guard interval $G>L$ available at the transmitter, after the N-point IDFT, a $G$-length cyclic prefix is added to $\tilde{\mathbf{s}}=\mathbf{F}^{H} \mathbf{s}$. The post-IDFT processing at the transmitter can then be represented by the matrix $\mathbf{C}_{c p}$. This leads us to the transmit signal representation

$$
\mathbf{x}=\mathbf{C}_{c p} \mathbf{F}^{H} \mathbf{s},
$$

At the receiver, instead of discarding the entire $\mathrm{CP}$, only the first $L$ samples of the received signal $\mathbf{r}$ are discarded. The $N+e_{t}$-length input $\tilde{\mathbf{y}}$ to the equalizer can then be represented as

$$
\widetilde{\mathbf{y}}=\mathbf{D}_{c p} \mathbf{H}_{t} \mathbf{C}_{c p} \mathbf{F}^{H} \mathbf{s}+\tilde{\mathbf{v}},
$$

where $\mathbf{D}_{c p}$ represents the removal of $L$-samples from CP. The resultant equivalent channel can be represented as a block

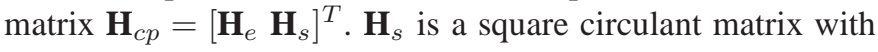
$N$ rows and corresponds to the equivalent matrix for full CPremoval. $\mathbf{H}_{e}$ is the time-domain channel matrix corresponding to the excess time present in CP. Similar to the case of cyclicprefixed ZP-OFDM $\widetilde{\mathbf{y}}$ can equivalently be represented as

$$
\widetilde{\mathbf{y}}=\left[\begin{array}{c}
\mathbf{y}_{e} \\
\widetilde{\mathbf{y}}
\end{array}\right]=\left[\begin{array}{c}
\mathbf{H}_{e} \\
\mathbf{H}_{s}
\end{array}\right] \mathbf{F}^{H} \mathbf{s}+\left[\begin{array}{c}
\widetilde{\mathbf{v}}_{e} \\
\widetilde{\widetilde{\mathbf{v}}}
\end{array}\right],
$$

We then process $\widetilde{\widetilde{\mathbf{y}}}$ with a $N$-point DFT while retaining $\mathbf{y}_{e}$ as is. Representing this operation by $\mathbf{J}_{c}$, we arrive at the following equivalent receive signal representation.

$$
\begin{aligned}
& \mathbf{y}=\mathbf{J}_{c} \widetilde{\mathbf{y}} \\
&=\left[\begin{array}{c}
\mathbf{H}_{e} \mathbf{F}^{H} \\
\mathbf{F H}_{s} \mathbf{F}^{H}
\end{array}\right] \mathbf{s}+\mathbf{v} \\
&=\mathcal{H}_{c} \mathbf{s}+\mathbf{v} \\
& \\
&{ }^{1}(A+B C D)^{-1}=A^{-1}-A^{-1} B\left(C^{-1}+D A^{-1} B\right)^{-1} D A^{-1}
\end{aligned}
$$


The MMSE estimator for the transmit signal $\mathbf{s}$ given $\mathbf{y}$ is then given by $\mathbf{R}_{s y} \mathbf{R}_{y y}^{-1}$ and the equalizer can be expressed as $\mathbf{G}_{c}=$ $\left(\mathcal{H}_{c}^{H} \mathcal{H}_{c}+\sigma_{v}^{2} / \sigma_{a}^{2} \mathbf{I}\right)^{-1} \mathcal{H}_{c}^{H}$ As in the case of cyclic-prefixed ZP-OFDM, here too, the equalizer can be implemented with low complexity by exploiting the structure of $\mathcal{H}_{c}$. By using excess time in $\mathrm{CP}$, the resultant equalizer is able to show a better BER performance. The equalizer however, has diversity order-1 in frequency selective channels due to the fact that $\mathbf{H}_{e}$ in this case loses rank if any fading coefficient of the multipath is zero.

\section{Numerical Results}

In this section we provide numerical results and compare diversity order with linear equalization at the receiver for frequency selective channels based on the outage probability analysis. For frequency selective channels, we compare the performance of CP-OFDM with MMSE equalization, ZPOFDM with MMSE-ZF equalizer which is known to achieve full diversity and the special case of ZP-OFDM introduced in Sec. III-B with the proposed equalizer. Monte-Carlo simulations are carried out for a fixed transmission rate over a 6-tap sample-spaced channel with equal power in all taps and each tap fading independently. The resulting SINR for an arbitrary, fixed subcarrier is computed. When this SINR is not able to support transmission at the predefined rate, channel is declared to be in outage. The slope of the outage probability curve is then used to estimate the diversity order of the receiver. In Fig. 1, we plot the outage probability for these three cases for the above simulation setup. We note that the slope of the outage probability for ZP-OFDM is greater than that of CP-OFDM system which is not surprising since CP-OFDM system, in the absence of any redundant precoding is not able to exploit multipath diversity present in the channel. The performance of cyclic prefixed ZP-OFDM with low complexity linear equalization for 3 different values of $e_{t}$ clearly indicates that as $e_{t}$ approaches $L$, the diversity of the system also approaches $L$. In other words, the system is able exploit the available excess time to provide multipath diversity with linear equalization. When $e_{t}=L$ the system thus enjoys full multipath diversity! In Fig. 2 we compare the performance of conventional MMSE equalization in CPOFDM that does not exploit excess time present in the $\mathrm{CP}$ with that of the equalizer that exploits this excess time. The BER is seen to improve, however, as mentioned earlier, the diversity order of the equalizer is 1 .

\section{CONCLUding Remarks}

To benefit from low complexity equalization and also exploit multipath diversity, CP-OFDM systems incur a loss in spectral efficiency due redundant linear precoding in addition to the loss due to the presence of an un-used guard interval. On the other hand, we show here that it is indeed possible to achieve full diversity with low complexity linear equalization in ZP-OFDM in frequency selective channels. Receivers exploiting excess time in CP show better BER performance with only a slight increase in complexity compared to conventional equalizers that do not exploit $\mathrm{CP}$ but do not provide any

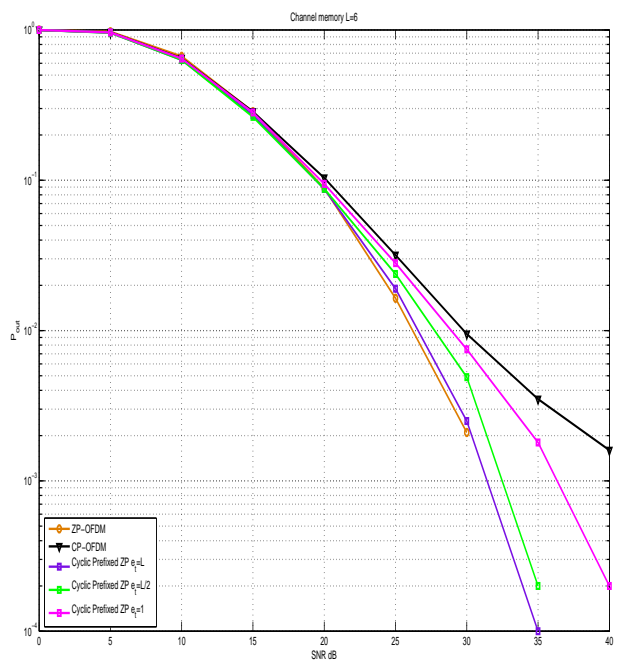

Fig. 1. Outage Probability results for frequency selective channels.

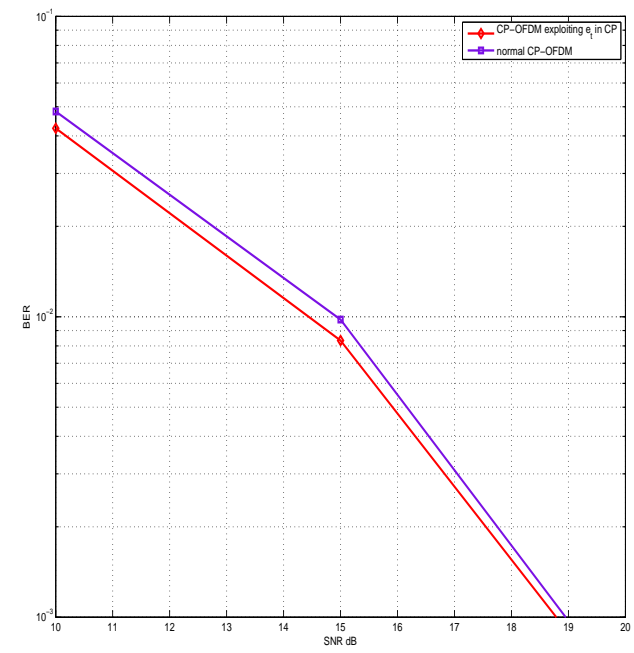

Fig. 2. BER comparison of CP-OFDM exploiting excess time in $\mathrm{CP}$.

diversity benefits. If the transmitter exploits channel length information, it can use a combination of $\mathrm{ZP}$ and $\mathrm{CP}$ to allow receivers to achieve varying diversity orders with linear equalizers depending on the excess time available at the transmitter.

\section{REFERENCES}

[1] Tepedelenlioglu. C., "Maximum multipath diversity with linear equalization in precoded OFDM systems," Information Theory. IEEE Transactions on, vol. 50, no. 1, pp. 232-235, Jan. 2004.

[2] B. Muquet, Zhengdao Wang, G.B. Giannakis, M. de Courville, and P Duhamel, "Cyclic prefixing or zero padding for wireless multicarrier transmissions?," Communications. IEEE Transactions on, vol. 50, no. 12, pp. 2136-2148, Dec 2002.

[3] Thomas Kailath, Linear Systems (Prentice-Hall Information and System Science Series), Prentice Hall, November 1979.

[4] C. Tepedelenlioglu and Qian Ma, "On the performance of linear equalizers for block transmission systems," Global Telecommunications Conference, 2005. GLOBECOM '05. IEEE, vol. 6, pp. 5 pp.-, Nov.-2 Dec. 2005. 